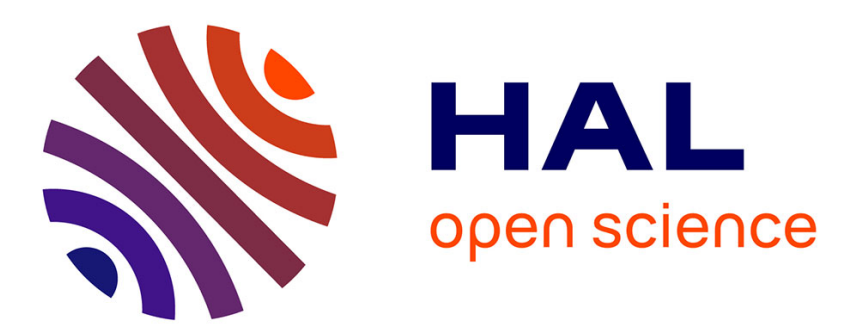

\title{
A 3-layered self-reconfigurable generic model for self-diagnosis of telecommunication networks
}

Serge Romaric Tembo Mouafo, Jean-Luc Courant, Sandrine Vaton

\section{To cite this version:}

Serge Romaric Tembo Mouafo, Jean-Luc Courant, Sandrine Vaton. A 3-layered self-reconfigurable generic model for self-diagnosis of telecommunication networks. IntelliSys 2015: SAI Intelligent Systems Conference, Nov 2015, London, United Kingdom. pp.25 - 34, 10.1109/IntelliSys.2015.7361080 . hal-01250445

\section{HAL Id: hal-01250445 \\ https://hal.science/hal-01250445}

Submitted on 26 Jan 2016

HAL is a multi-disciplinary open access archive for the deposit and dissemination of scientific research documents, whether they are published or not. The documents may come from teaching and research institutions in France or abroad, or from public or private research centers.
L'archive ouverte pluridisciplinaire HAL, est destinée au dépôt et à la diffusion de documents scientifiques de niveau recherche, publiés ou non, émanant des établissements d'enseignement et de recherche français ou étrangers, des laboratoires publics ou privés. 


\section{A 3-Layered Self-Reconfigurable Generic Model For Self-Diagnosis of Telecommunication Networks}

\author{
Serge Romaric Tembo \\ Orange Labs \\ 2 Avenue Pierre Marzin \\ 22300 Lannion, France \\ Email: serge.tembo@orange.com
}

\author{
Jean-Luc Courant \\ Orange Labs \\ 2 Avenue Pierre Marzin \\ 22300 Lannion, France \\ Email: jeanluc.courant@orange.com
}

\author{
Sandrine Vaton \\ Telecom Bretagne \\ 655 Avenue du Technopole \\ 29200 Brest, France \\ Email: sandrine.vaton@telecom-bretagne.eu
}

\begin{abstract}
The dynamic and distributed nature of telecommunication networks makes complex the design of model-based approaches for network fault diagnosis. Most model-based approaches assume the prior existence of the model which is reduced to a static image of the network. Such models become rapidly obsolete when the network changes. We propose in this paper a 3-layered self-reconfigurable generic model of fault diagnosis in telecommunication networks. The Layer 1 of the model is an undirected graph which models the network topology. Network behavior, also called fault propagation, is modeled in Layer 2 using a set of directed acyclic graphs interconnected via the Layer 1. We handle uncertainties of fault propagation by quantifying strengths of dependencies between Layer 2 nodes with conditional probability distributions estimated from network generated data. Layer 3 is the junction tree representation of the loopy obtained Layer 2 Bayesian networks. The junction tree is the diagnosis computational layer since exact inference algorithms fail on loopy bayesian networks. This generic model embeds intelligent self-reconfiguration capabilities in order to track some changes in network topology and network behavior. These self-reconfiguration capabilities are highlighted through some example scenarios that we describe. We apply this 3-layered generic model to carry out active self-diagnosis of the GPONFTTH access network. We present and analyze some experimental diagnosis results carried out by running a Python implementation of the generic model.
\end{abstract}

Keywords-Self-diagnosis; Self-reconfiguration; fault propagation; Bayesian network; Probabilistic Inference; GPON; FTTH.

\section{INTRODUCTION}

Reliability, robustness, availability, accessibility are the most important requirements that telecommunication networks should guarantee. As the design of network architectures, network management also became a central issue for telecommunication operators, which have triggered significant researches about autonomic networking. The main goal of autonomic networking is to automate as much as possible numerous tedious operations of network management like fault management.

Fault diagnosis is a central aspect of network fault management [27]. Traditionally, fault diagnosis has been performed manually by an expert or a group of experts experienced in managing communication networks [28]. However, the development of telecommunication networks has increased the size and complexity of their architectures. Fault diagnosis has become too complex for humans, who can keep track of only few hypotheses in their reasonings. Humans need a long training to fully master their network segment [6]. Automating fault diagnosis is critical in large scale telecommunication networks. A network fault or failure is a root cause of one or multiple network anomalies observed in the form of alarms or parameters outside of the standard. Alarms or symptoms are external manifestations of failures [8]. A telecommunication network is naturally a distributed system, a fault occurred spreads, triggering other faults and alarms which in turn trigger further faults and alarms. The consequence of both fault and alarm propagation is that a single root cause may result in a complex and distributed pattern of subsequent failures and their corresponding alarms [6]. This is especially true when multiple faults propagate simultaneously.

Fault diagnosis also called alarm correlation or root cause diagnosis [8], [13], [29], isolates the most probable set of faults based on their external manifestations. It is a process of searching intelligible explanations to observed alarms. Fault diagnosis is a complex process due to the non-deterministic nature of fault propagation phenomenon. A single fault may generate multiples alarms and a single alarm may be triggered by several faults.

The fault diagnosis problem was addressed in the past two decades and numerous techniques have been proposed. Expert systems [20], [25] encode specialized reasonings on specific diagnosis tasks in computer applications. Model-based approaches [4] develop reasonings based on an explicit representation of the network. The techniques based on machine learning algorithms like artificial neural networks [22], [1], probabilistic networks [28], [21] and case-based reasoning [14] infer diagnosis based on past experiences.

A brief description and discussion of related work on fault diagnosis as well as our objectives is presented in section 2 . We describe and formalize in section 3, our contribution: a generic three layered self-reconfigurable probabilistic model for telecommunication networks fault diagnosis. In section 4, we focus on self-reconfiguration capabilities of the generic model by showing how this model can be turn into an intelligent autonomous system for self-diagnosis purposes. In section 5, we present an application of the generic model to fault diagnosis of the FTTH (Fiber To The Home) access networks based on GPON (Gigabit capable Passive Optical Network). In section 6, some experimental diagnosis results are presented and analysed with respect to the GPON-FTTH network. We conclude and present future works in section 7. 


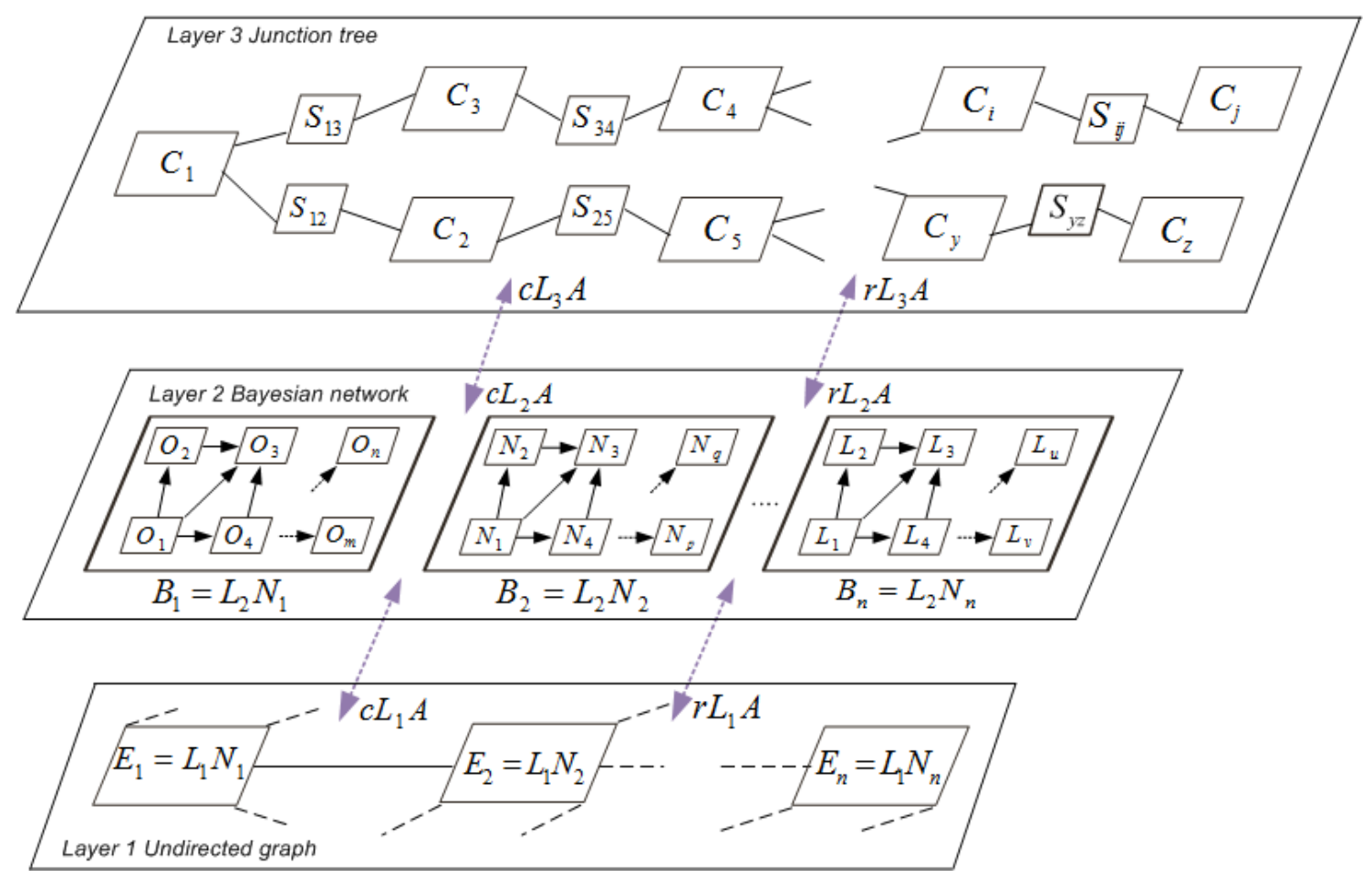

Fig. 1: The 3-Layered Generic Model.

\section{RELATED WORKS}

Early self-diagnosis approaches were called expert systems [20], [25]. An expert diagnosis system attempts to infer the cause of a problem from symptoms recognized in sensor data [6]. It is a problem-solving software that embodies specialized reasonings on narrow diagnosis tasks usually performed by a trained skilled human called expert. The specialized reasonings can be formalized with rules, list of facts, logic predicates, etc. The inference engines are commonly based on forwardbackward [15] chaining algorithms. However, expert systems mainly suffer of brittleness. The system fails when it faces a novel problem that comes out of its expertise.

Model-based diagnosis approaches [5], [10], [9], [11], [12], [23] develop reasonings based on formal and explicit representation of network structure and network behavior. Network structure describes the network architecture. Network behavior describes the process of alarm propagation and alarm correlation [7]. Network structure and network behavior are then modeled [4]. The obtained model is the support of reasoning algorithms which must be designed. The modelbased approach is easy to deploy and is appropriate for a largescale network if information on network resources is available [16]. It has the ability to deal with novel problems although its performances degrade in this case.

Model-based approach seems natural when relationships between objects are graph-like and easy to obtain [27]. The model can be designed in a modular or incremental fashion facilitating updates as new knowledge about the network is acquired. However, it is quite difficult to build a model close enough to the structural and functional reality of the network while maintaining a high level of abstraction to make the model independent of the various engineering techniques implemented in telecommunication networks. In addition, the model built is reduced to a static image of the network and becomes rapidly obsolete when the network changes.

In order to deal with the difficulty to obtain and selfmaintain an accurate model of fault propagation in large scale telecommunication networks, we propose in this paper a generic 3-layered self-reconfigurable probabilistic model for self-diagnosis of telecommunication networks. The model integrates two fields: a decision field and an artificial learning field. The decision field is based on Bayesian probabilistic reasoning [21] in order to deal with uncertainties of fault propagation process. The artificial learning [3] field brings selfreconfiguration capabilities to the generic model in order to deal with the dynamic nature of telecommunication networks. The generic model has capabilities to compute diagnosis decisions and to automatically learn changes in network topology and network behaviour.

\section{A LAYERED SELF-RECONFIGURABLE PROBABILISTIC MODEL}

We focus in this section on the description and the formalism of the 3-layered generic model which can be applied to any distributed system for self-diagnosis purposes.

\section{A. Description of the generic model}

As in any distributed system, in telecommunication networks, faults typically propagate between related system components. There is also some cases where a fault can propagate only inside a system component without affecting the state of neighbor system components. We call this, local fault 


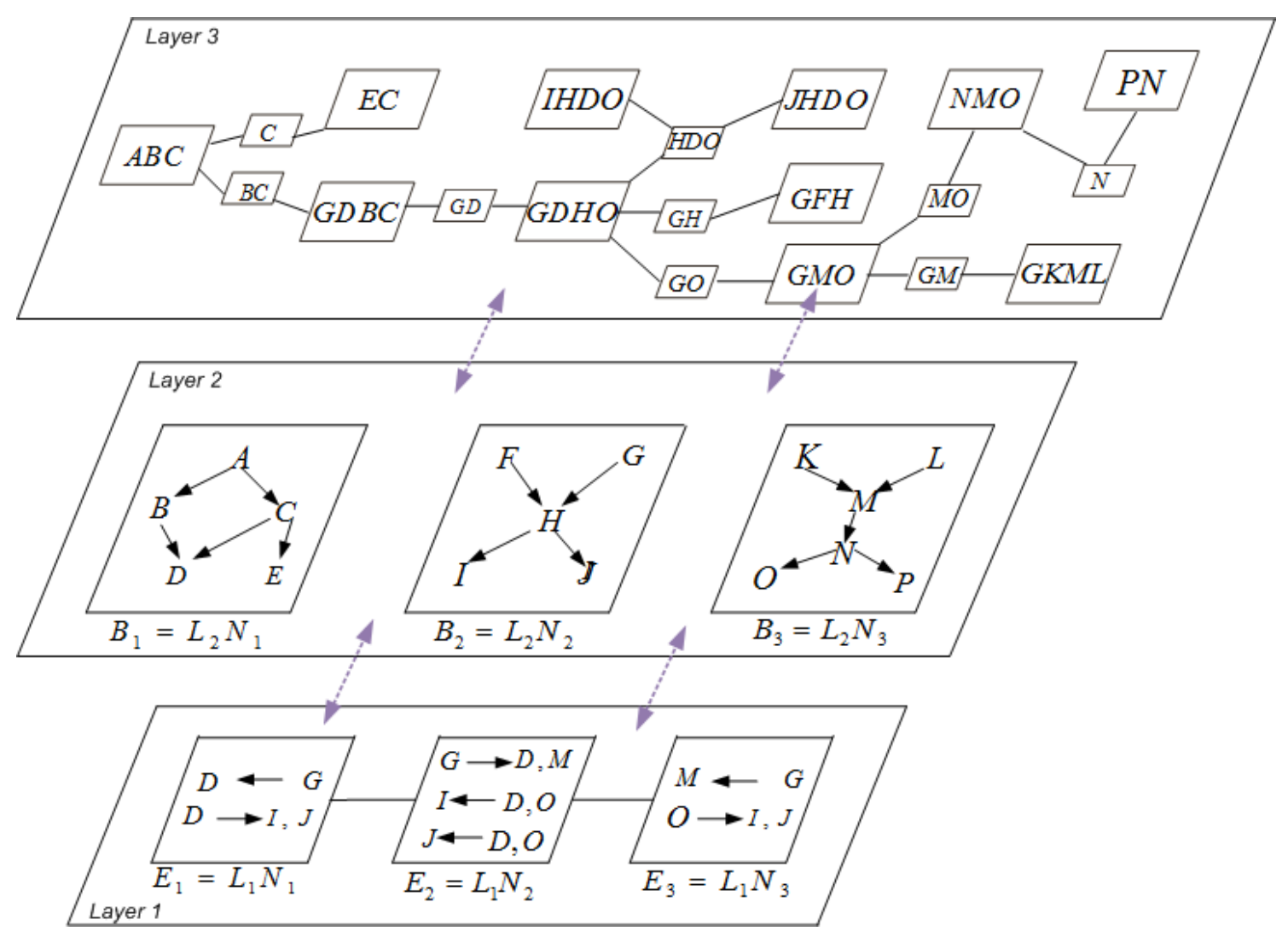

Fig. 2: A simple example of the 3-Layered Generic Model.

propagation. Obviously, distributed fault propagation is more recurrent in communication systems than their local counterpart. But keep in mind that a fault can also spread locally to a network component. A local fault propagation model is made of local dependencies which are relationships between faults (root causes), intermediate faults, counters, scalar parameters and alarms which can be observed on a network component.

The generic model that we propose clearly considers local and distributed fault propagation by separating network topology modeling and fault propagation modeling. Note that this separation brings important properties to the generic model: modularity and extensibility which facilitates selfreconfiguration. The generic model has three layers. Layer 1 models the network topology. Layer 2 models fault propagation. Layer 3 is a simplification of layer 1 and layer 2 .

The layer 1 models the network topology and distributed fault propagation between related network components. Layer 1 is an undirected graph whose nodes represent network components and edges represent bidirectional links between them (see Figure 1). Any node of this layer is a Directed Acyclic Graph (DAG) which embeds distributed dependencies between a network component and its neighbors. These distributed dependencies model fault propagation between the related network components. The layer 1 of the generic model is an undirected graph of DAGs, i.e, a layer 1 node is seen as a subset of layer 2 nodes linked via distributed dependencies. In Figure 1, layer 1 represents the topology of a network of $n$ components $E_{1}, E_{2}, \ldots, E_{n}$. The network component $E_{1}$ is linked to one neighbor $E_{2}$. The network components $E_{1}$ and $E_{2}$ are each DAGs containing distributed dependencies that carry fault propagation between them. For example, in Figure 2, each of the components $E_{1}$ and $E_{3}$ embeds distributed dependencies with its neighboor $E_{2}$. The component $E_{2}$ embeds distributed dependencies with its neighboors $E_{1}$ and $E_{3}$. In Figure 1, the roles of layer 1 agents $c L_{1} A, r L_{1} A$, layer 2 agents $c L_{2} A, r L_{2} A$ and layer 3 agents $c L_{3} A, r L_{3} A$ will be discussed later in the paper.

The layer 2 is a set of DAGs. Each DAG models local fault propagation inside a network component, i.e. a layer 1 node. In Figure 1, a layer 2 DAG $B_{i}$ models local fault propagation on network component $E_{i}$. The DAGs of layer 2 are interconnected via layer 1 nodes. In order to deal with uncertainties of fault propagation phenomenon, each DAG can be transformed into a Bayesian network by quantifying strength of dependencies with Conditional Probability Distributions (CPD). A CPD quantifies the degree of influence that a subset of nodes of a DAG has on their common successor node. In Figure 1, Layer 2 contains $n$ belief networks interconnected via Layer 1 . The belief network $B_{i}$ models local fault propagation inside the network component $E_{i}$. We call Layer 2 node a variable of any belief network.

Note that the union of DAGs of layer 2 and DAG of each layer 1 node gives one large DAG which models fault propagation in the entire network. But the separation between network topology modeling and network behavior (fault propagation) modeling breaks down this large DAG in many 
interconnected parts. So the design of layer 1 and layer 2 implements this separation and brings us the modularity and extensibility properties necessary to provide easy selfreconfiguration capabilities to the generic model.

The distributed nature of a telecommunication network introduces mutual dependencies between related network components. This means that distributed undirected loops are unavoidable between some layer 2 nodes. In addition, undirected loops may also appear in local fault propagation inside a network component. Note that, carrying out a message passing inference algorithm like the sum-product algorithm [21] at layer 2 will fail due to local and distributed undirected loops. In order to deal with local and distributed undirected loops between layer 2 nodes, we have built a third layer upon layer 2. The layer 3 is the junction tree representation [15], [16], [26] of layer 1 and layer 2.

Note that layer 1 and layer 2 are useful for learning field of the generic model since they provide modularity and extensibility properties useful for easy self-reconfiguration. Layer 3 is useful for decision field of the generic model since inference can be easily done in this layer regardless of network topology complexity at layer 1 and network behavior complexity at layer 2 .

\section{B. Formalism of the generic model}

We formalize in this subsection the three layers of the generic model. We first define a local dependency and a distributed dependency. Let $B_{i}$ and $B_{j \neq i}$ be two distinct layer 2 Directed Acyclic Graphs (DAGs). A local dependency on $B_{i}$ is a tuple $(u, v)$ such that $u$ and $v$ belong to the set of nodes of $B_{i}$. A distributed dependency between $B_{i}$ and $B_{j \neq i}$ is a tuple $(u, v)$ such that $u$ belongs to the set of nodes of $B_{i}$ and $v$ belongs to the set of nodes of $B_{j}$. For example, in Figure 2, $(B, D)$ is a local dependency which is part of the local fault propagation model on network component $E_{1}$. $(G, D)$ is a distributed dependency between the related network components $E_{1}$ and $E_{2}$.

Layer 1 denoted by $L_{1}$ Graph is an undirected graph of DAGs, $L_{1}$ Graph $=\left(L_{1}\right.$ Nodes, $L_{1}$ Edges $)$. $L_{1}$ Nodes is the set of $n$ layer 1 nodes or network components considered, $L_{1}$ Nodes $=\left\{L_{1} N_{1}, L_{1} N_{2}, \ldots, L_{1} N_{n}\right\} . L_{1}$ Edges is the set of bidirectional links between layer 1 nodes.

Each layer 1 node $L_{1} N_{i}$ is a DAG defined as follows: $L_{1} N_{i}=\left(L_{1} V_{i}, L_{1} E_{i}\right) . L_{1} V_{i}$ is the subset of layer 2 nodes embedded in layer 1 node $L_{1} N_{i}$. Each layer 2 node belonging to $L_{1} V_{i}$ is part to at least one distributed dependency. $L_{1} E_{i}$ is the set of distributed dependencies between $L_{1} N_{i}$ and its $k_{i} \geq 1$ layer 1 neigboor nodes. $L_{1} V_{i}$ is defined as follows:

$$
L_{1} V_{i}=N_{i} \bigcup\left(\cup_{j=1}^{k_{i}} N_{j}^{i}\right)
$$

where, $N_{i} \subset L_{2} V_{i}, N_{j}^{i} \subset L_{2} V_{j}, j \in\{1, \ldots, n\} \backslash\{i\}$ such that $\forall u \in N_{i}, \exists v \in N_{j}^{i} \mid u \in p a(v) \vee v \in p a(u)$. We suppose that layer 1 node $L_{1} N_{i}$ has $k_{i}$ neighboors. $L_{2} V_{i}$ is the subset of layer 2 nodes belonging to layer $2 \mathrm{DAG} L_{2} N_{i}$ above layer 1 node $L_{1} N_{i}$ (see Figure 1). The layer 2 DAG $L_{2} N_{i}$ models local fault propagation on layer 1 node $L_{1} N_{i}$. For example, in Figure 2, $L_{1} N_{2}=\left(L_{1} V_{2}, L_{1} E_{2}\right)$ such that $L_{1} V_{2}=N_{2} \cup N_{1}^{2} \cup N_{3}^{2}$, where the subset $N_{2}=\{G, I, J\}, N_{1}^{2}=\{D\}$ and $N_{3}^{2}=$
$\{M, O\} . L_{1} E_{2}$ is the set of distributed dependencies between the component $L_{1} N_{2}$ and its neighboors $L_{1} N_{1}$ and $L_{1} N_{3}$. $L_{1} E_{2}=\{(G, D),(G, M),(D, I),(O, I),(D, J),(O, J)\}$

Layer 2 is a set of $n$ DAGs. Each DAG $L_{2} N_{i}=$ $\left(L_{2} V_{i}, L_{2} E_{i}\right)$ models local fault propagation on network component $L_{1} N_{i} . L_{2} E_{i}$ is the set of local dependencies between layer 2 nodes belonging to $L_{2} V_{i}$.

Layer 3 is the junction tree representation of the large bayesian network $G$ obtained by combining layer 1 and layer 2 as follows: $G=\bigcup_{i=1}^{n}\left[L_{2} N_{i} \cup L_{1} N_{i}\right]$. Layer 3 is constructed in three steps. The moralization [17] of $G$ and the triangulation [17] of the obtained moralized graph lead to a chordal graph or clique graph. The moralization consist to disorient edges of the graph $G$ and adding a disoriented edge between each couple of parents of each node of $G$. The triangulation consist to add an edge to every cycle of the moralized graph whose length exceeds 3 . The maximal weight spanning tree of the obtained clique graph is guaranteed to be a junction tree. The weight is the size of the intersection between adjacent cliques, i.e, the number of layer 2 nodes shared by adjacent cliques. We call layer 3 node, a clique $C_{i}$ of the junction tree. As every junction tree, layer 3 satisfies the running intersection property which ensures that, the intersection $C_{i} \cap C_{j}$ is a subset of every clique and separator on the path between two cliques $C_{i}$ and $C_{j}$. For example, in Figure 2, $G=C_{G D B C} \cap C_{G K M L}: G$ belongs to the clique $C_{G M O}$ and the separators $S_{G O}$ and $S_{G M}$ which form the path between the cliques $C_{G D B C}$ and $C_{G K M L}$.

\section{Diagnosis computations of the generic model}

The layer 3 is an equivalent of layer 1 and layer 2. This means that layer 3 is sufficient to compute diagnosis decisions using for example the well known HUGIN exact inference algorithm [15] on a junction tree. In Figure 1, layer 3 nodes $C_{i}, C_{j}, S_{i j}$ are respectively the cliques $C_{i}$ and $C_{j}$ of the junction tree and their common separator $S_{i j}$. A layer 3 node is a compound variable of some layer 2 nodes. For example, in Figure 2, layer 3 node $A B C$ is a compound variable of Layer 2 nodes $A, B$, and $C$. Layer 3 is initialized by associating a potential to each layer 3 node. At initialization, a clique $C_{i}$ has the potential $\phi_{C_{i}}$ and a separator $S_{i j}$ has the potential $\phi_{S_{i j}}$ as follows:

$$
\phi_{C_{i}}=\prod_{X \in \text { Layer } 2, X \in C_{i}, p a(X) \subset C_{i} \vee p a(X)=\emptyset} P(X \mid p a(X))
$$

And

$$
\phi_{S_{i j}}=1
$$

We note $p a(X)$, the parent set of layer 2 node $X$. The potential $\phi_{C_{i}}$ of a layer 3 node $C_{i}$ represents the joint conditional probability of layer 2 nodes that compose it (see equation 2). For example, in Figure 2: $\phi_{C_{A B C}}=P(B \mid A) \cdot P(C \mid A) \cdot P(A)$, $\phi_{C_{G D B C}}=P(D \mid B, C, G) \cdot P(G)$ and $\phi_{S_{B C}}=1$.

The diagnosis decisions computed at layer 3 are based on evidences propagation on a junction tree. Assume $C_{i}, C_{j}$ to be neighbooring layer 3 nodes with their common separator $S_{i j}$ (see Figure 1). The potential $\phi_{C_{i}}$ is updated when some layer 2 nodes belonging to clique $C_{i}$ are observed. This evidence propagates from clique $C_{i}$ to clique $C_{j}$ towards their separator $S_{i j}$ as follows: updating the potential of the separator $S_{i j}$ by the marginalization of equation 4 , and updating the potential 
of the clique $C_{j}$ by the product of equation 5 . The notation $C_{i} \backslash S_{i j}$ reprensents the layer 2 nodes of the clique $C_{i}$ which does not belong to the separator $S_{i j}$.

$$
\begin{aligned}
\phi_{S_{i j}}^{*} & =\sum_{C_{i} \backslash S_{i j}} \phi_{C_{i}}^{*} \\
\phi_{C_{j}}^{*} & =\phi_{C_{j}} \frac{\phi_{S_{i j}}^{*}}{\phi_{S_{i j}}}
\end{aligned}
$$

We say that the clique $C_{j}$ absorbs evidences from $C_{i}$ [15] or that the clique $C_{i}$ brings evidences to $C_{j}$. Note that, update operations of clique potentials and separator potentials are done in two recursive stages. The first stage called collect is initiated by collecting evidences from observed nodes to the root nodes. The second stage is initiated by distributing evidences from the root nodes to leave nodes. Collection of evidences to a clique $C_{i}$ is done by collecting evidences to all the children of $C_{i}$ followed by absorption of evidences from each child. Similarly, distribution of evidences from a clique amounts to bring evidences to each child followed by distribution of evidences from the child.

Note that, the update operations of potentials of layer 3 nodes are made by an agent called computing Layer 3 Agent $c L_{3} A$. The $c L_{3} A$ updates the potential of each layer 3 node when it receives some evidences (observed layer 2 nodes) from its counterpart of layer 2 called $c L_{2} A$. When $c L_{2} A$ receives updated layer 3 node potentials, it computes the marginals (beliefs) of layer 2 nodes. These two agents may communicate using a simple mechanism like shared memory through an interface between layer 2 and layer 3 that we call L2-L3 Interface (see Figure 3).

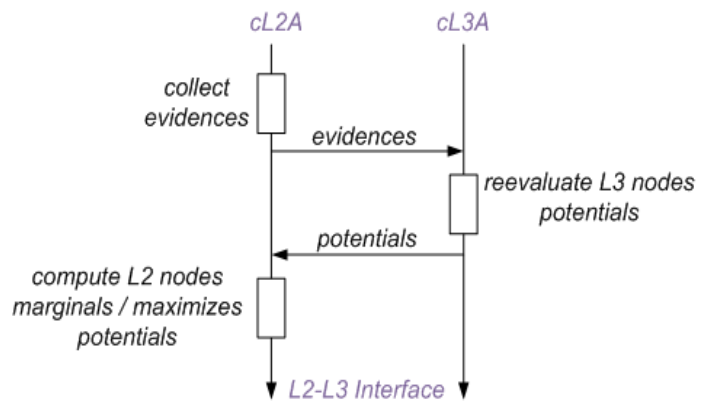

Fig. 3: Communication between layer 2 and layer 3 for beliefs updating of layer 2 nodes.

The marginal $P(X \mid e)$ of a layer 2 node $X$ consistent with evidences $e$, is computed from the updated potential $\phi_{C_{i}}^{*}$ of any layer 3 node $C_{i}$ such that $X \in C_{i}: P(X \mid e)=\sum_{C_{i} \backslash X} \phi_{C_{i}}^{*}$. Note that, after updating the potential of all layer 3 nodes, the intersection property ensures coherence between updated potentials, i.e, the marginal of a layer 2 node $X$ is the same regardless of the clique $C_{i}$ on which the marginalization operation is made. The most probable state of the layer 2 node $X$ that is consistent with evidences, is one that has the highest probability.

Note that, there is an alternative approach to find the diagnosis without performing summation operations on updated potentials. With this approach, the diagnosis $r^{*}$, is computed from the most probable explanation $w^{*}$, of evidences as follows: $w^{*}=\bigcup_{C \in \text { Layer } 3} w_{C}^{*}$ such that $\phi_{C}\left(w_{C}^{*}\right)=$ $\max _{w_{C}} \phi_{C}\left(w_{C}\right)$, where $w_{C}$ is a configuration of layer 2 nodes belonging to layer 3 node $C$ and $w_{C}^{*}$ maximizes the potential $\phi_{C}$ of $C$. The diagnosis $r^{*}$ is the most probable configuration of layer 2 root nodes defined by $r^{*}=w^{*} \backslash i^{*}$, where $i^{*}$ is the most probable configuration of non root layer 2 nodes consistent with evidences.

\section{Self-Reconfiguration CAPABilities of the GENERIC MODEL}

In this subsection, we illustrate the potential of the generic model to self-reconfigure in order to track changes in network topology and network behavior (how faults propagate). Each of the three layers of the generic model has a reconfiguration agent which communicates by shared memory with its counterpart of the adjacent layers.

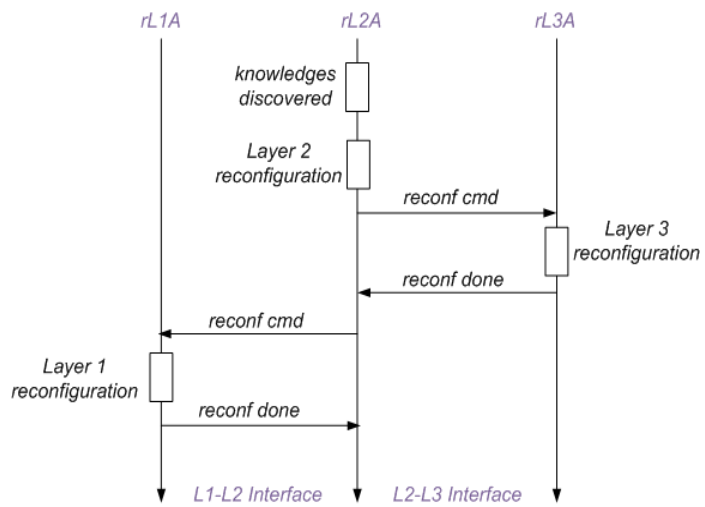

Fig. 4: Self-reconfiguration process initiated at layer 2.

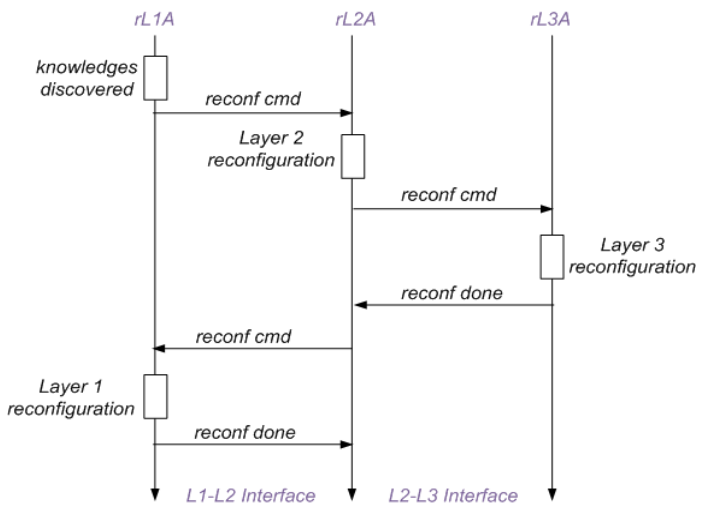

Fig. 5: Self-Reconfiguration process initiated at layer 1.

The reconfiguration Layer 2 Agent $r L_{2} A$ is an artificial learning [3] system which implements knowledge discovery and data mining algorithms from a tremendous amount of network generated data. This agent must have the ability to discover new local and distributed dependencies, new states of layer 2 nodes to consider, new network statistical knowledges which require a new estimation of conditional probabilities of some layer 2 nodes. When the $r L_{2} A$ discovers some new important knowledge about network behavior, it reconfigures the layer 2 of the model and sends a reconfiguration command 
(reconf $\mathrm{cmd}$ ) to the reconfiguration Layer 3 Agent $r L_{3} A$ and to the reconfiguration Layer 1 Agent $r L_{1} A$ (see Figure 4). The message reconf done is an acknowledgement of the command reconf $\mathrm{cmd}$.

Note that $r L_{2} A$ will send a reconfiguration command to $r L_{1} A$ only if the new knowledge learned concerns distributed dependencies. Indeed, local dependencies are unknown at layer 1. Note also that it is the type of the new knowledge learned at layer 2 that will permit $r L_{3} A$, after receiving a reconfiguration command from $r L_{2} A$, to know if it should reconfigure or rebuild the layer 3 . For example, if the conditional probability distribution of a layer 2 node changes, the $r L_{3} A$ needs only to raise an event notifying the computing Layer 3 Agent $c L_{3} A$, to recompute the initial potential of all layer 3 nodes which this layer 2 node belongs to. If new local and/or distributed dependencies are learned, the $r L_{3} A$ must rebuild the layer 3 .

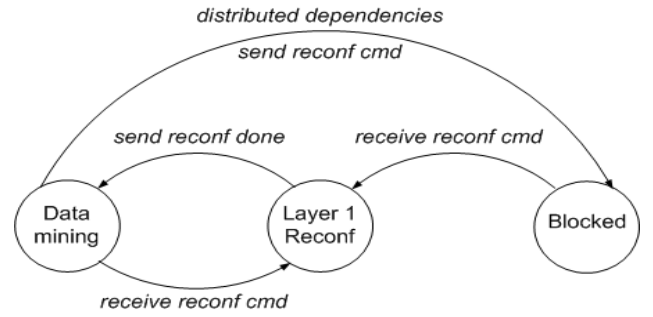

Fig. 6: State machine of the layer 1 reconfiguration agent.

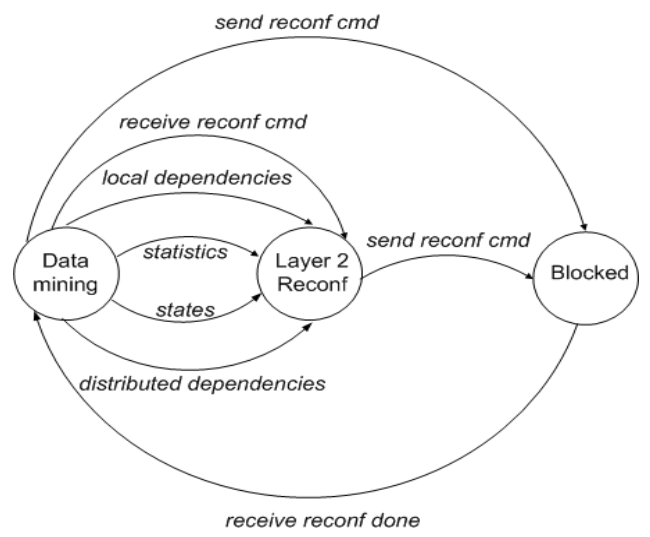

Fig. 7: State machine of the layer 2 reconfiguration agent.

The function of the reconfiguration Layer 1 Agent $r L_{1} A$ is to track network topology changes like adding or removal of a network component. The $r L_{1} A$ can be an automatic network topology discovery system [2], [24] or a system with an administration interface. This interface allows experts to remove a network component from the model or to specify and add a new network component to the model. The specification of a new network component includes: the name of this component, its neighbor components which already exist in the model, its local dependencies (local fault propagation model) and the distributed dependencies with each neighbor.

Note that any change in network topology requires the reconfiguration of the three layers of the model. The Figure 5 illustrates this reconfiguration process initiated by the layer 1 reconfiguration agent. The dynamic behaviour of the $r L_{1} A$ is modelled by a 3 -states machine (see Figure 6). Initially, the model is at equilibrium, i.e, there is no running reconfiguration process. So the $r L_{1} A$ is in Data mining state, i.e, it waits for specification of a new network component by a network expert, it searchs for new knowledges about network topology from data collected by carried out active measures on network. When, the $r L_{1} A$ discovers new knowledges about network topology, e.g, distributed dependencies, it does not reconfigure the layer 1 immediately. It sends a reconf $c m d$ to the $r L_{2} A$ and transits to the Blocked state. In Blocked state, the $r L_{1} A$ waits for the completion of upper layers reconfiguration before entering in Layer 1 Reconf state. The $r L_{1} A$ transits from Blocked state to Layer 1 Reconf state when it receives a reconf cmd from $r L_{2} A$. In the case where the $r L_{2} A$ discovers new distributed dependencies, the $r L_{1} A$ transits from Data mining to the Layer 1 Reconf state when it receives a reconf cmd from $r L_{2} A$, and returns to the Data mining state when it completes the layer 1 reconfiguration. The completion of the layer 1 reconfiguration yields a new equlibrium state of the model.

Layer 1 reconfiguration adds or removes new layer 1 node (network component) to/from the model. For adding a node for example, the new layer 1 node embeds distributed dependencies with its layer 1 neighboor nodes which already exist in the model. The $r L_{1} A$ sends a reconf $c m d$ to $r L_{2} A$ (see Figure 5), waits for the $r L_{2} A$ completes the layer 2 reconfiguration and adds the distributed dependencies to the neighboor nodes of the new layer 1 node. To reconfigure the layer 2, the $r L_{2} A$ builds a layer 2 Bayesian network which models local fault propagation on the new network component. The $r L_{2} A$ notifies the computing Layer 2 Agent $c L_{2} A$ to recompute the conditional probability distribution of each layer 2 nodes having new distributed dependencies learned or specified at layer 1 . Remark that only the reconfiguration agent of layer 2 takes a reconfiguration decision based on knowledges learned from network generated data. The other reconfiguration agents $\left(r L_{1} A\right.$ and $\left.r L_{3} A\right)$ wait for a reconf cmd from $r L_{2} A$ before starting a reconfiguration process. Therefore, the $r L_{2} A$ is the coordinator of our multi-agent based self-reconfigurable model.

The Figure 7 specifies the dynamic behaviour of the layer 2 reconfiguration agent using a 3-states machine. Initially, the $r L_{2} A$ is in Data mining state, i.e, it searchs for new knowledges about network behaviour from tremendous amount of data generated by the network components. When the $r L_{2} A$ learns new local dependencies, new distributed dependencies, new states of a layer 2 node to consider or new important statistical knowledges, it transits to the Layer 2 Reconf state in order to integrate the new knowledges learned to the layer 2 of the generic model. The $r L_{2} A$ should trigger the reconfiguration of adjacents layer by sending a reconfiguration command to the reconfiguration agent of these layers. The Blocked state implements synchronisation between two successive reconfiguration processes, i.e, the $r L_{2} A$ waits for the running reconfiguration process of the model completes before triggering another process. Doing so, the completeness and consistency of the model remain guaranteed after the integration of new learned knowledges to the generic model. 


\section{Application of the Generic Layered Model to GPON-FTTH SELF-DIAGNOSIS}

We show in this section how the generic model previously proposed can be applied to perform self-diagnosis of FTTH (Fiber To The Home) access networks based on GPON (Gigabit capable Passive Optical Network) [19], [18]. For now, we used prior knowledges acquired from ITU-T standards [19], [18] of the GPON-FTTH network to build a 3-layered model of this network. We plan to transform this GPON-FTTH network model into an autonomous system by implementing self-reconfiguration capabilities of the generic model. The Figure 8 depicts the architecture of the GPON-FTTH network.

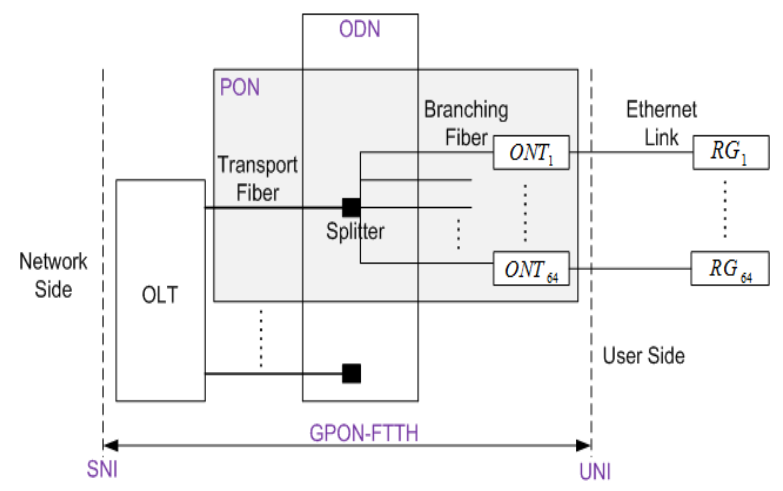

Fig. 8: GPON-FTTH network architecture.

The GPON-FTTH access network has two main network components. The Optical Line Termination $(O L T)$ is located on operator side. The Optical Network Termination $(O N T)$ is located on customer side. The $O L T$ and $O N T$ are connected through an Optical Distribution Network $(O D N)$. The $O D N$ is the optical infrastructure made of fibers and passive components like splitters. A Passive Optical network $(P O N)$ is a point-to-multipoint link inside the $O D N$. A $P O N$ has a tree-like topology which connects an $O L T$ with a maximum of 64 ONTs (see Figure 8). Each $O N T$ is connected to a $R G$ (Residential Gateway) via an Ethernet link.

Since there is no interaction between PONs in $O D N$, and all PONs have the same behavior, we modeled one single $P O N$. This model can be replicated to any $P O N$ of a GPONFTTH access network. All ONTs connected to the same PON temporally share the upstream optical channel of the PON. The downstream channel of the $P O N$ is a secured broadcasting channel. The composition of Figure 9 and Figure 10 forms the application of the generic model for modeling the topology and behavior of a PON of the GPON-FTTH access network. The obtained model has two layer 1 nodes (OLT, ONT) and some layer 2 nodes are vectors in order to consider the tree-like topology of a $P O N$.

\section{A. Upstream modeling}

The Figure 9 depicts the layer $1 O L T$ node and its layer 2 local dependencies. Distributed dependencies embedded in layer $1 O L T$ node are also depicted. We have three types of layer 2 nodes in Figure 9: faults or root causes, intermediate faults and alarms. The root causes are highlighted in Figure 9.
The transport optical fiber of the PON denoted by Fiber $_{T}(O K, A T, B R)$ can take three states. The state $O K$ means that there is no transmissions anomaly on this fiber. The states $A T$ and $B R$ mean respectively that fiber experiences high attenuation or that fiber is broken. The temperature of $O L T$ denoted by $T_{O L T}^{c}$, is a continous variable that we discretize. The power supply of $O L T$ denoted by $A l t_{O L T}$ which can be faulty or not.

The node FaultyONT denotes an $O N T$ which transmits upstream signal outside of its granted time slot, which may conflict with data sent by other ONTs on the PON and cause data disruption for a random set of $O N T$, making the PON unusable. A FaultyONT can cause a Drift of Windows $D O W$. $O L T$ raises a $D O W[i]$ alarm when an $O N T[i]$ transmits signal beyond the slot time allocated to it. See ITU-T G984.3 [19], [18] for more details.

The Software Version $S W V[i]$ alarm means that there is an incompatibility between the Image Operating System (IOS) of ONT[i] and those of the OLT. The node ONTConfMis (ONT Configuration Mistake) denotes a configuration error during $O N T$ provisioning.

The $O L T$ transmitted power $T x_{O L T}$ is regulated by the bias current $I_{O L T}$. This leads to the local dependency $I_{O L T} \longrightarrow$ $T x_{O L T}$. The $O L T$ received power $R x_{O L T}[i]$ from an $O N T_{i}$ depends of the $O L T$ voltage $V_{O L T}$ and the state of the transport fiber Fiber $_{T}$.

The $O L T$ received power of $O N T_{i}$ also depends of the state of the branching fiber denoted by Fiber $D B[i]$ and the transmitted power of this $O N T_{i}$ denoted by $T x_{O N T}[i]$. Note two local dependencies $\left(\right.$ Fiber $\left._{T} \longrightarrow R x_{O L T}, V_{O L T} \longrightarrow R x_{O L T}\right)$ and two distributed dependencies (Fiber $D B \longrightarrow R x_{O L T}$, $\left.T x_{O N T} \longrightarrow R x_{O L T}\right)$. Remark in Figure 9 that the distributed dependencies are part of edges of the layer $1 O L T$ node which is a DAG as designed in the generic model.

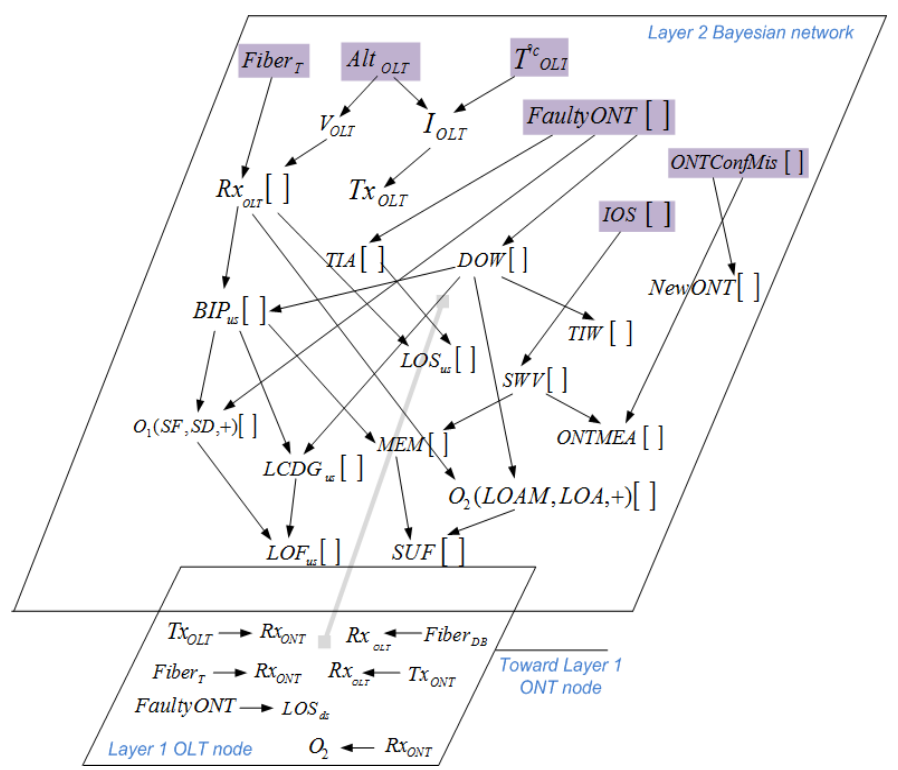

Fig. 9: Faults and alarms propagation raised by an $O L T$.

The Bit Interleaving Parity denoted by BIPus $[i]$ is computed from the Bit Error Rate BER of an upstream data 
transmission between an $O N T_{i}$ and $O L T$. A poor upstream signal reception can cause bit errors leading to the local dependency $R x_{O L T} \longrightarrow$ BIPus. Upstream transmission bit errors impact the quality of signal received by $O L T$ which may raised some alarms related to signal quality like $S D$ (Signal Degraded), $S F$ (Signal Fail), $L C G D$ (Loss of GEM Channel Delineation) and $M E M$ (Message Error Message). See ITU-T G984.3 [19] recommendation for more details.

\section{B. Downstream modeling}

The Figure 10 depicts the layer $1 O N T$ node and its layer 2 local dependencies. The root causes Alt $_{O N T}[i], T_{O N T}^{c}[i]$ and Fiber $D B[i]$ respectively denote the power supply, the temperature and the branching fiber of $O N T[i]$.

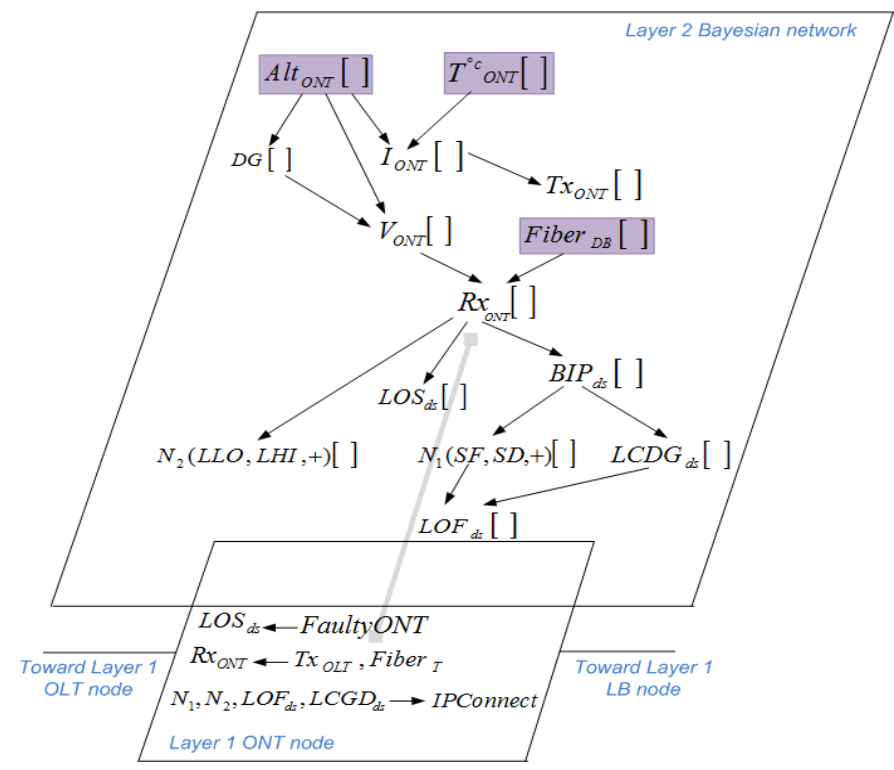

Fig. 10: Faults and alarms propagation raised by an $O N T$.

When the received power $R x_{O N T}[i]$ of an $O N T_{i}$ is less than a preconfigured minimum threshold, this $O N T$ raises the (Level Low) $L L O[i]$ alarm. The (Level High) LHI $[i]$ alarm is raised by $O N T_{i}$ when $R x_{O N T}[i]$ is greater than a preconfigured maximum threshold. For simplicity and because $L L O$ and $L H I$ alarms can not be observed simultaneously, we have considered them as the states of the layer 2 node called $N_{2}(L L O, L H i,+)$. The state denoted by + means that there is no $L L O$ or $L H I$ alarm observed. The received power $R x_{O N T}[i]$ depends of the voltage $V_{O N T}[i]$, the state of the branching fiber Fiber $D B[i]$, the state of the transport fiber Fiber $_{T}$ of the $P O N$ and the transmitted power $T x_{O L T}$ of $O L T$. Note the local dependencies $V_{O N T} \longrightarrow R x_{O N T}$, FiberDB $\longrightarrow R x_{O N T}$ and the distributed dependencies $\mathrm{Tx}_{\mathrm{OLT}} \longrightarrow R x_{\mathrm{ONT}}, \mathrm{Fiber}_{T} \longrightarrow R x_{\mathrm{ONT}}$.

Now, suppose we need to extend this model by adding another layer 1 node: a Residential Gateway $R G$ node for example (see Figure 8). A $R G$ is a home network component that provides services to customers. It is not a GPON-FTTH network component, but adding it to the model will bring to the model the ability to correlate faults and alarms of the GPONFTTH network with malfunctions of customer services. For example, in Figure 10, we can make such correlation with the distributed dependency $N_{1}, N_{2}, L O F_{d s}, L C G D_{d s} \longrightarrow$ IPConnect, where IPConnect denotes the Internet access service provided by a $R G$. To add the layer $1 R G$ node, we will need only to specify and quantify uncertainties of its local dependencies and distributed dependencies with the layer 1 ONT node, which already exists in the model.

\section{EXPERIMENTAL RESULTS OF THE GPON-FTTH NETWORK SELF-DIAGNOSIS}

We present and analyse in this section the network fault diagnosis results carried out by our application and implementation of the generic model to perform active self-diagnosis of GPON-FTTH access network. The experiments are performed on a GPON-FTTH testbed on which we have considered a $P O N$ with two $O N T s$ named $O N T_{1}$ and $O N T_{2}$. This experimental testbed allows us to emulate faults and to collect alarms raised by the GPON-FTTH network components. We also read, if availaible, the values of counters $B I P_{u s}, B I P_{d s}$ and scalar parameters $R x_{O L T}, R x_{O N T}, T x_{O L T}, T x_{O N T}$, voltages $V_{O L T}, V_{O N T}$, bias current $I_{O L T}, I_{O N T}$, temperatures $T_{O L T}^{c}, T_{O N T}^{c}$ of $O L T$ and of the two ONTs connected to the PON considered. Each table from Table I to Table VII shows the beliefs of Layer 2 root cause nodes computed by our Python implementation of the decision field of the generic model based on evidences observed and decribed in the title of each table.

We start the evaluation of the designed probabilistic fault propagation model for GPON-FTTH self-diagnosis by ckecking if inference on this model returns no fault when no alarm is observed on the $P O N$ and when counters and scalar parameters of $O L T$ and of the two ONTs are nominal. The Table I presents the result of this test and shows that the positive state in bold, i.e, the good working state of each layer 2 root cause node is the most probable state.

TABLE I: No Fault: no observed alarm, counters and scalar parameters are known and are nominal for both $O N T_{1}$ and $O N T_{2}$.

\begin{tabular}{|c|c|c|}
\hline Faults & States & Beliefs \\
\hline Fiber $_{T}$ & {$[\mathbf{O K}, A T, B R]$} & {$[\mathbf{0 . 9 9}, 6 . \mathrm{e}-03,7 . \mathrm{e}-12]$} \\
\hline AltOLT & {$[\mathbf{O K}, D F]$} & {$[0.99,4 . e-07]$} \\
\hline Faulty $O N T_{1}$ & {$[+f t o$, -fto $]$} & {$[0.007, \mathbf{0 . 9 9 3}]$} \\
\hline Faulty $\mathrm{ONT}_{2}$ & {$[+f t o$, -fto $]$} & {$[0.014, \mathbf{0 . 9 8 6}]$} \\
\hline$I O S_{1}$ & {$[\mathbf{O K}, D F]$} & {$[\mathbf{0 . 9 8 0}, 0.020]$} \\
\hline$I \mathrm{OS}_{2}$ & {$[\mathbf{O K}, D F]$} & {$[\mathbf{0 . 9 8 0}, 0.020]$} \\
\hline ONTConfMis $s_{1}$ & {$[+o c m,-\mathbf{o c m}]$} & {$[0.002, \mathbf{0 . 9 9 8}]$} \\
\hline $\mathrm{ONTConfMis}_{2}$ & {$[+o c m,-\mathbf{o c m}]$} & {$[0.002, \mathbf{0 . 9 9 8}]$} \\
\hline FiberD $B_{1}$ & {$[\mathbf{O K}, A T, B R]$} & {$[\mathbf{0 . 9 1}, 8 . e-02,9 . e-07]$} \\
\hline FiberDB $B_{2}$ & {$[\mathbf{O K}, A T, B R]$} & {$[\mathbf{0 . 9 1}, 8.2-02,9 . e-07]$} \\
\hline$A l t O N T_{1}$ & {$[\mathbf{O K}, D F]$} & {$[0.99,1 . e-07]$} \\
\hline $\mathrm{AltON}_{2}$ & {$[\mathbf{O K}, D F]$} & {$[\mathbf{0 . 9 9}, 1 . \mathrm{e}-07]$} \\
\hline
\end{tabular}

The Table II shows that the $O N T_{1}$ is diagnosed to be faulty when it loses upstream and downstream communication with $O L T$ although the optical signal between them is not degraded (no alarm related to signal quality is observed). Note the missing values of counters and scalar parameters of $O N T_{1}$ since the GPON-FTTH network management system has no way to get these values from MIB (Management Information Base) of $O N T_{1}$. These missing values useful to compute the most probable diagnosis are inferred by the probabilistic model by using its conditional probability distributions. The 
loss of upstream and downstream communication between $O L T$ and $O N T_{1}$ may also be due to the cut of the branching fiber of $O N T_{1}$. But this is a very rare event in reality since infrastructures of telecommunication operators always protect optical fibers from possible cuts. That is why the most probable explanation of a bidirectional loss of communication is a faulty $O N T$. However, other observations in addition to LOSus 1 and $L O S d s_{1}$ alarms may change this explanation.

TABLE II: Loss of communication between $O L T$ and $O N T_{1}$ with no alarm related to signal quality: alarms $L O S d s_{1}, L O S u s_{1}$ are observed, counters and scalars are unknown for $O N T_{1}$ but known and nominal for $O N T_{2}$.

\begin{tabular}{|l|l|l|}
\hline Faults & States & Beliefs \\
\hline Fiber $T$ & {$[O K, A T, B R]$} & {$[0.99,6 . e-03,1 . \mathrm{e}-11]$} \\
\hline Faulty $O N T_{1}$ & {$[$ +fto, - fto $]$} & {$[\mathbf{0 . 9 9 8}, 0.002]$} \\
\hline Faulty $O N T_{2}$ & {$[+$ fto,- fto $]$} & {$[0.014,0.986]$} \\
\hline Fiber $D B_{1}$ & {$[O K, A T, B R]$} & {$[0.91,8 . e-02,1 . e-06]$} \\
\hline Fiber $D B_{2}$ & {$[O K, A T, B R]$} & {$[0.91,8 . e-02,9 . e-07]$} \\
\hline
\end{tabular}

TABLE III: Loss of communication between $O L T$ and $O N T_{1}$ with alarms related to signal quality: alarms $L O S d s_{1}, L O S u s_{1}, L C G D u s_{1}, L C G D d s_{1}, S D u s_{1}, S D d s_{1}$ are observed, counters and scalars are unknown for $O N T_{1}$ but known and nominal for $\mathrm{ONT}_{2}$.

\begin{tabular}{|l|l|l|}
\hline Faults & States & Beliefs \\
\hline Fiber & {$[O K, A T, B R]$} & {$[0.94,5 . \mathrm{e}-02,1 . \mathrm{e}-7]$} \\
\hline Faulty $O N T_{1}$ & {$[+$ fto,- fto $]$} & {$[0.422,0.578]$} \\
\hline Faulty $O N T_{2}$ & {$[+$ fto, - fto $]$} & {$[0.014,0.986]$} \\
\hline Fiber $D B_{1}$ & {$[O K, \mathbf{A T}, B R]$} & {$[0.409, \mathbf{0 . 5 7 7}, 0.014]$} \\
\hline Fiber $D B_{2}$ & {$[O K, A T, B R]$} & {$[0.92,7 . \mathrm{e}-02,1 . \mathrm{e}-06]$} \\
\hline
\end{tabular}

TABLE IV: Attenuation of the branching fiber of $O N T_{1}$ with known counters and scalar parameters: alarms $S D d s_{1}, S D u s_{1}$ are observed, variation of counters BIPus $s_{1}$ upstream and $B I P d s_{1}$ downstream, low level of upstream received power $R x O L T_{1}$ and downstream received power $R x O N T_{1}$. Counters and scalar parameters of $O N T_{2}$ are known and are nominal.

\begin{tabular}{|l|l|l|}
\hline Faults & States & Beliefs \\
\hline Fiber $_{T}$ & {$[O K, A T, B R]$} & {$[0.91,8 . e-02,1 . \mathrm{e}-11]$} \\
\hline${\text { Faulty } O N T_{1}}_{1}$ & {$[+$ fto,- fto $]$} & {$[1 . e-06,0.999]$} \\
\hline Faulty $O N T_{2}$ & {$[+$ fto,- fto $]$} & {$[0.014,0.986]$} \\
\hline Fiber $D B_{1}$ & {$[O K, \mathbf{A T}, B R]$} & {$[7 . e-02, \mathbf{0 . 9 2}, 2 . \mathrm{e}-06]$} \\
\hline Fiber $D B_{2}$ & {$[O K, A T, B R]$} & {$[0.92,7 . e-02,1 . e-06]$} \\
\hline
\end{tabular}

TABLE V: Attenuation of the branching fiber of $O N T_{1}$ with unknown counters and scalar parameters of $O N T_{1}$ : alarms $S D d s_{1}, S D u s_{1}$ are observed, counters and scalar parameters of $O N T_{2}$ are known and nominal.

\begin{tabular}{|l|l|l|}
\hline Faults & States & Beliefs \\
\hline Fiber $_{T}$ & {$[O K, A T, B R]$} & {$[0.93,6 . \mathrm{e}-02,1 . \mathrm{e}-09]$} \\
\hline Faulty $O N T_{1}$ & {$[+$ fto, - fto $]$} & {$[0.082,0.918]$} \\
\hline Faulty $O N T_{2}$ & {$[+$ fto,- fto $]$} & {$[0.014,0.986]$} \\
\hline Fiber $D B_{1}$ & {$[O K$, AT, $B R]$} & {$[2 . e-01, \mathbf{0 . 7 6}, 1 . \mathrm{e}-04]$} \\
\hline Fiber $D B_{2}$ & {$[O K, A T, B R]$} & {$[0.92,7 . e-02,1 . e-06]$} \\
\hline
\end{tabular}

In Table III, the observation of alarms notifying optical signal degradation, brings additional information to the model and changes the decision of the inference algorithm. The $O N T_{1}$ is no longer diagnosed to be Faulty but its branching fiber experiences important attenuation leading to communication loss with OLT. Remark in Table III that, contrary to the Table II, the belief that $O N T_{1}$ is faulty decreases in favor of increasing the belief that the branching fiber of $O N T_{1}$ experiences important attenuation.

In Table IV, the most probable diagnosis is the attenuation of the branching fiber of $O N T_{1}$ when SD (Signal Degraded) alarms are raised for $O N T_{1}$ with its counters and scalar parameters known and non-nominal (upstream and downstream BIP variation, low level upstream and downstream receive power). If for some reasons, the management system of GPON-FTTH network fails to get the values of counters and scalars of $O N T_{1}$, the model is always able to take the best decision although in this case, the belief in the branching fiber attenuation of $O N T_{1}$ decreases slightly (from 0.92 to 0.76 , see Table V).

TABLE VI: Attenuation of the branching fiber of $O N T_{1}$ and $O N T_{2}$ with known counters and scalars of $O N T_{1}$ and $O N T_{2}$ : alarms $S D u s_{1}, S D d s_{1}, S D u s_{2}, S D d s_{2}$ are observed, variation of BIPus, BIPds for both ONTs, low level of upstream and downstream received power for both $O N T s$.

\begin{tabular}{|l|l|l|}
\hline Faults & States & Beliefs \\
\hline Fiber $_{T}$ & {$[O K, \mathbf{A T}, B R]$} & {$[0.41, \mathbf{0 . 5 8}, 1 . \mathrm{e}-09]$} \\
\hline${\text { Faulty } O N T_{1}}$ & {$[+$ fto, - fto $]$} & {$[1 . \mathrm{e}-04,0.999]$} \\
\hline Faulty $O N T_{2}$ & {$[+$ fto, - fto $]$} & {$[5 . \mathrm{e}-04,0.999]$} \\
\hline Fiber $D B_{1}$ & {$[O K, \mathbf{A T}, B R]$} & {$[0.49, \mathbf{0 . 5 0}, 1 . \mathrm{e}-05]$} \\
\hline Fiber $D B_{2}$ & {$[O K, \mathbf{A T}, B R]$} & {$[0.49, \mathbf{0 . 5 0}, 1 . e-05]$} \\
\hline
\end{tabular}

Now, if we observe signal degradation between $O L T$ and $O N T_{1}$, and between $O L T$ and $O N T_{2}$ (see Table VI), the probabilistic model more likely believes that it is the transport fiber of the $P O N$ which experiences attenuation rather than each of the two branching fibers. Remark in Table VI that the probability of the transport fiber attenuation is 0.58 . The probability of attenuation of each branching fiber is 0.50. The difference between these two probabilities is not very significant. Therefore, we may tend to believe that the model has trouble to take the best decision between the transport fiber attenuation or branching fibers attenuation. But, if we compute the joint probability of the two branching fibers attenuation $(0.5 \times 0.5=0.25)$, we remark that the difference between this joint probability and the probability of the transport fiber attenuation is now very important. This result enforces the belief that it is the transport fiber of the PON which experiences attenuation. Note that the joint probability of the two branching fibers is the product of the probabilities of the branching fibers since they are independent. In fact the Markov Blanket $M B\left(\right.$ Fiber $\left.D B_{i}\right)$ of the variable Fiber $D B_{i}$ is defined by the union of the following sets: the parent set $P A_{i}$ of FiberD $B_{i}$, the children set $C H_{i}$ of FiberDB $B_{i}$ and the set $O P_{i}$ of other parents of children of Fiber $D B_{i} . M B_{i}=P A_{i} \cup C H_{i} \cup O P_{i}$, where $P A_{i}=\emptyset$ (the empty set) since $F$ iber $D B_{i}$ is a layer 2 root cause node. The set $C H_{i}=\left\{R x_{O L T_{i}}, R x_{O N T_{i}}\right\}$ and the set $O P_{i}=\left\{T x_{O N T_{i}}, V_{O N T_{i}}, V_{O L T}, T x_{O L T}, F_{i b e r}\right\}$. The branching fibers Fiber $D B_{1}$ and Fiber $D B_{2}$ are independent each other because: FiberDB $B_{1} \notin M B\left(\right.$ Fiber $\left.D B_{2}\right)$ and Fiber $D B_{2} \notin M B\left(\right.$ Fiber $\left.D B_{1}\right)$.

The Table VII illustrates the case when a downstream loss of communication between $O L T$ and $O N T_{1}$ is observed, i.e, the alarm $L O S d s_{1}$ is raised and the GPON-FTTH network 
TABLE VII: Loss downstream communication between $O L T$ and $O N T_{1}: L O S d s_{1}$ alarm is observed, counters and scalar parameters of $O N T_{1}$ are unknown, but known and nominal for $O N T_{2}$.

\begin{tabular}{|l|l|l|}
\hline Faults & States & Beliefs \\
\hline Fiber $_{T}$ & {$[O K, A T, B R]$} & {$[0.92,7 . e-02,3 . e-11]$} \\
\hline Faulty $O N T_{1}$ & {$[+$ fto,- fto $]$} & {$[0.094,0.906]$} \\
\hline Faulty $O N T_{2}$ & {$[+$ fto,- fto $]$} & {$[0.014,0.986]$} \\
\hline Fiber $D B_{1}$ & {$[O K$, AT,$B R]$} & {$[8 . e-02, \mathbf{0 . 9 1}, 4 . e-06]$} \\
\hline Fiber $D B_{2}$ & {$[O K, A T, B R]$} & {$[0.92,7 . e-02,1 . e-06]$} \\
\hline
\end{tabular}

management system does not read the values of counters and scalar parameters of $O N T_{1}$. Note that, this is a one way loss of communication. Contrary to a bidirectional loss of communication as illustrated in Table II, $O N T_{1}$ is not diagnosed to be faulty. The most probable diagnosis computed by the model is the branching fiber downstream attenuation of $O N T_{1}$, i.e, only the downstream optical channel between $O L T$ and $O N T_{1}$ experiences attenuation.

\section{CONCLUSION}

We have presented in this paper a generic 3-layered probabilistic model of fault diagnosis of telecommunication networks. The generic model embeds two fields: a decision field and an artificial learning field. The generic model has capabilities to compute diagnosis decisions and to automatically learn changes in network topology and network behaviour.

We have shown how this generic model can be applied to design a probabilistic model-based approach of fault diagnosis of GPON-FTTH access network. We have presented and analyzed some network fault diagnosis results carried out by running a Python implementation of the decision field of the generic model. The results obtained are very consistent with experiments carried out on a GPON-FTTH network testbed.

We plan to transform this GPON-FTTH network faults diagnosis model into an autonomous system by implementing self-reconfiguration capabilities of the generic model. Another perspective is to bring more intelligence to the reconfiguration agent of layer 3 by designing a novel paradigm allowing it to take into account new dependencies learned to the lower layers without rebuilding all the layer 3. Doing so, scalability and reconfiguration efficiency of the generic model will increase.

\section{REFERENCES}

[1] J. R. A. Goel and P. Sadayappan. Towards a 'neural' architecture for abductive reasoning. IEEE International Conference on Neural Networks, pages 681-688, 1998.

[2] M. N. Beacon. A hierarchical network topology monitoring system based in IP multicast. Services Management in Intelligent Networks, no. 1960 in Lecture Notes in Computer Science, pages 169-180, 2000.

[3] A. Cornuéjols and L. Miclet. Apprentissage Artificiel, Concepts et Algorithmes. EYROLLES, 2013.

[4] R. Gardner and D. Harle. Alarm correlation and network fault resolution using the kohonen self-organising map. Global Telecommunications Conference (GLOBECOM 1997), pages 1398-1402, 1997.

[5] B. Gruschke. Integrated event management: Event correlation using dependency graphs. A.S. Sethi (Ed.), Ninth International. Workshop on Distributed Systems: Operations and Management, University of Delaware, Newark, DE, 87:130-141, October 1998.
[6] C. Hounkonnou. Active self-diagnosis in telecommunication networks. $\mathrm{PhD}$ Thesis, European University of Brittany, University of Rennes 1, INRIA, ISTIC, French, 2013.

[7] G. Jakobson and M. Weissman. Real-time telecommunication network management: extending event correlation with temporal constraints. In Proceedings of the fourth international symposium on Integrated network management IV, pages 290-301, 1995.

[8] G. Jakobson and M. Weissmani. Alarm correlation. IEEE Network, 7(6):52-59, 1993.

[9] J. Jordaan and M. Paterokl. Event correlation in heterogeneous networks using the osi management framework. H.G. Hegering, Y. Yemini (Eds.), Integrated Network Management III, North-Holland, Amsterdam, 36:683-695, 1993.

[10] S. C. K. Houck and A. Finkel. Towards a practical alarm correlation system. A.S. Sethi, F. Faure-Vincent, Y. Raynaud (Eds.), Integrated Network Management IV, Chapman and Hal, London, 86:226-237, 1995.

[11] S. Kätker. A modeling framework for integrated distributed systems fault management. C. Popien (Ed.), Proc. IFIP/IEEE Internat. Conference on Distributed Platforms, Dresden, Germany, pages 187-198, 1995.

[12] S. Kätker and K. Geihs. A generic model for fault isolation in integrated management systems. Journal of Network and Systems Management, 5(2):109-130, 1997.

[13] I. Katzela and M. Schwartz. Schemes for fault identification in communication networks. IEEE Transactions on Networking, 3(6), 1995.

[14] L. Lewis. A case-based reasoning approach to the resolution of faults in communication networks. Integrated Network Management, pages 671-682, 1993.

[15] A. L. Madsen and F. V. Jensen. Lazy propagation: A junction tree inference algorithm based on lazy evaluation. Artificial Intelligence, 113:203-245, 1999.

[16] M. Miyazawa and K. Nishimura. Scalable root cause analysis assisted by classified alarm information model based algorithm. 7th International Conference on Network and Service Management, pages 1-4, 2011.

[17] P. Naim, P.-H. W., P. Leray, O. Pourret, and A. Becker. Réseaux Bayésiens. EYROLLES, 2008.

[18] T. S. S. of ITU. G.977.1 Recommendation. ITU-T, 2003.

[19] T. S. S. of ITU. G.984.3 Recommendation. ITU-T, 2008.

[20] L. PAU. Survey of expert systems for fault detection, test generation and maintenance. Expert Systems, 3:100-110, April 1986.

[21] J. Pearl. Probabilistic reasoning in intelligent systems: networks of plausible inference. Morgan Kaufmann, 1988.

[22] J. C. R.J. Patton and T. Siew. Fault diagnosis in nonlinear dynamic systems via neural networks. International Conference on Control, 2:1346-1351, 1994.

[23] M. P. S. Kätker. Fault isolation and event correlation for integrated fault management. A. Lazar, R. Sarauo, R. Stadler (Eds.), Integrated Network Management V, Chapman and Hall, London, 60:583-596, 1997.

[24] D. C. S. Ramanathan and S. Neal. Auto-discovery capabilities for service management: An isp case study. Journal of Network and Systems Management, 8(4):457-482, 2000.

[25] W. T. Scherer and C. White. A survey of expert systems for equipment maintenance and diagnostics. Fault detection and reliability: knowledge based and other approaches. Pergamon Press, pages 3-18, 1987.

[26] D. S. Stefen Laurizen. Local computations with probabilities on graphical structures and their application to expert systems. Journal of the Royal Statistical Society, Series B, 50(2):157-224, 1988.

[27] M. Steinder and A. S. Sethi. A survey of fault localization techniques in computer networks. Science of Computer Programming, 53:165-194, January 2004.

[28] M. Steinder and A. S. Sethil. Multi-layer fault localization using probabilistic inference in bipartite dependency graph. University of Delaware, Newark, DE, Technical Report, 2001.

[29] S. A. Yemini, S. Kliger, E. Mozes, Y. Yemini, and D. Ohsie. High speed and robust event correlation. IEEE Communications Magazine, 34(5):82-90, April 1996. 